Pacific Journal of Mathematics

ON THE TRANSFORMATION OF FOURIER COEFFICIENTS 


\title{
ON THE TRANSFORMATION OF FOURIER COEFFICIENTS OF CERTAIN CLASSES OF FUNCTIONS II
}

\author{
KENNETH F. ANDERSEN
}

\begin{abstract}
If $\left\{a_{\nu}\right\}_{1}^{\infty}$ is the sequence of Fourier cosine coefficients of a function in the space $L^{p}, 1 \leq p<\infty$, a Theorem of Hardy states that the sequence of averages $\left\{\left(\sum_{j=1}^{\nu} a_{j}\right) / \nu\right\}_{1}^{\infty}$ arise as Fourier cosine coefficients of a function also in $L^{p}$. Analogous results for the sequence $\left\{\sum_{j=\nu}^{\infty} a_{j} / j\right\}_{1}^{\infty}$ were obtained by Bellman. In this paper, sufficient conditions on the non-negative weight function $\omega(x)$ are given in order that the weighted Lebesgue space $L^{p}(\omega(x) d x)$ may replace the spaces $L^{p}$ in the Theorems of Hardy and Bellman.
\end{abstract}

1. Introduction and statement of results. Let $\left\{a_{\nu}\right\}_{0}^{\infty}$ denote the sequence of Fourier cosine coefficients of the integrable function $f(x)$, that is,

$$
a_{\nu}=\frac{2}{\pi} \int_{0}^{\pi} f(x) \cos \nu x d x, \quad \nu=0,1,2, \ldots,
$$

and let $A_{0}=A_{0}^{\prime}=a_{0}$,

$$
A_{\nu}=\frac{1}{\nu} \sum_{j=1}^{\nu} a_{j}, \quad A_{\nu}^{\prime}=\sum_{j=\nu}^{\infty} a_{j} / j, \quad \nu=1,2, \ldots
$$

G. H. Hardy [4] has shown that if $f(x)$ belongs to $L^{p}(0, \pi)$ for some $p, 1 \leq p<\infty$, then $\left\{A_{\nu}\right\}_{0}^{\infty}$ is the sequence of Fourier cosine coefficients of a function $F(x)$, also in $L^{p}(0, \pi)$; R. Bellman [3] proved the analogous statement for $\left\{A_{\nu}^{\prime}\right\}_{1}^{\infty}$, except that now $p$ satisfies $1<p \leq \infty$. These results have been generalized by several authors in various directions. In particular, we [1] have recently characterized those function spaces $L^{\sigma}(0, \pi)$, given by a rearrangement invariant metric $\sigma$, that may replace the $L^{p}(0, \pi)$ spaces in the Theorems of Hardy and Bellman.

In this paper, we consider a generalization in a direction complementary to that of the rearrangement invariant spaces. We shall consider here weighted spaces of functions $L^{p}(\omega)=\left\{f: \int_{0}^{\pi}|f(x)|^{p} \omega(x) d x=\|f\|_{p, \omega}^{p}<\right.$ $\infty$, giving conditions on the non-negative weight function $\omega(x)$ which ensure that $L^{p}(\omega)$ may replace the (unweighted) spaces $L^{p}$ in the Theorems of Hardy and Bellman. We suppose throughout, when required, that functions $f$ and weights $\omega$ defined initially on $(0, \pi)$ are defined on $(-\infty, \infty)$ by the requirements of evenness on $(-\pi, \pi)$ and $2 \pi$-periodicity. 
Since we shall be concerned with the Fourier cosine coefficients of an $f$ in $L^{p}(\omega)$ we shall have to have $L^{p}(\omega) \subset L^{1}$. Hölder's inequality

$$
\begin{aligned}
& \int_{0}^{\pi}|f(x)| d x \\
& \quad \leq \begin{cases}\left(\int_{0}^{\pi}|f(x)|^{p} \omega(x) d x\right)^{1 / p}\left(\int_{0}^{\pi} \omega(x)^{-1 /(p-1)} d x\right)^{1 / p^{\prime}} & \text { if } 1<p<\infty, \\
\left(\int_{0}^{\pi}|f(x)| \omega(x) d x\right)(\underset{(0, \pi)}{\operatorname{ess} \cdot \sup 1 / \omega(x))} & \text { if } p=1,\end{cases}
\end{aligned}
$$

and its converse show that this is equivalent to the requirement that

$$
\begin{cases}\int_{0}^{\pi} \omega(x)^{-1 /(p-1)} d x<\infty & \text { if } 1<p<\infty, \\ \operatorname{ess} \cdot \sup 1 / \omega(x)<\infty & \text { if } p=1 .\end{cases}
$$

Further, since we wish $L^{p}(\omega)$ to contain the constant functions, we assume that $\int_{0}^{\pi} \omega(x) d x<\infty$. Thus, $\theta(u)$ given by

$$
\begin{aligned}
& \theta(u)=\theta(\omega, p ; u) \\
& = \begin{cases}\sup _{a}\left(\int_{a}^{a+u} \omega(x) d x\right)^{1 / p}\left(\int_{a}^{a+u} \omega(x)^{-1 /(p-1)} d x\right)^{1 / p^{\prime}} & \text { if } 1<p<\infty, \\
\sup _{a}\left(\int_{a}^{a+u} \omega(x) d x\right)(\underset{(a, a+u)}{\operatorname{ess} \cdot \sup 1 / \omega(x))} & \text { if } p=1,\end{cases}
\end{aligned}
$$

is finite for all $u>0$, and we assume throughout that $\omega$ satisfies the additional mild condition $\int_{0}^{\delta} \theta(u) d u / u<\infty$ for some $\delta>0$. The restrictions we have placed on $\omega$ thus far may then be summarized by the equivalent, single requirement that

$$
\int_{0}^{2 \pi} \theta(\omega, p ; u) \frac{d u}{u}<\infty .
$$

For example, $\omega(x)=|\sin x|^{\alpha}$ satisfies (1.1) if and only if

$$
\begin{cases}-1<\alpha<p-1 & \text { if } 1<p<\infty \\ -1<\alpha \leq 0 & \text { if } p=1\end{cases}
$$

More generally, the well known and important Muckenhoupt class $A_{p}$ of weights, defined by the requirement that $\theta(u) \leq C u$, satisfy (1.1). Of course, a weight satisfying (1.1) need not satisfy the $A_{p}$ condition; for example, with $p=2, \omega(x)=x^{-1}(\log (\pi / x))^{-2}$ satisfies $(1.1)$ but not the $A_{2}$ condition.

We can now state our results. 
THEOREM 1. Let $p=1$. Suppose $\omega$ satisfies (1.1) and there is a constant $C$ such that for almost all $t, 0<t<\pi$,

$$
\int_{0}^{t} \omega(x) d x \leq C t \omega(t) .
$$

If $\left\{a_{\nu}\right\}_{0}^{\infty}$ is the sequence of Fourier cosine coefficients of an $f \in L^{1}(\omega)$ then $\left\{A_{\nu}\right\}_{0}^{\infty}$ is the sequence of Fourier cosine coefficients of a function $F$ also in $L^{1}(\omega)$; moreover, there is a constant $c$ independent of $f$ such that $\|F\|_{1, \omega} \leq$ $c\|f\|_{1, \omega}$.

Since $\omega$ clearly satisfies (1.2) if $\omega$ satisfies the $A_{1}$ condition, we have the following result.

Corollary 1. If $\omega$ is an $A_{1}$ weight then the conclusion of Theorem 1 holds.

THEOREM 2. Let $1<p<\infty$. Suppose $\omega$ satisfies (1.1) and that there is a constant $C$ such that for some $\varepsilon>0$

$$
\left(\int_{0}^{r} \omega(x) d x\right)^{1 / p}\left(\int_{r}^{\pi}\left(\frac{r}{x}\right)^{\varepsilon} x^{-p^{\prime}} \omega(x)^{-1 /(p-1)} d x\right)^{1 / p^{\prime}} \leq C
$$

holds for all $0<r<\pi$. If $\left\{a_{\nu}\right\}_{0}^{\infty}$ is the sequence of Fourier cosine coefficients of an $f \in L^{p}(\omega)$ then $\left\{A_{\nu}\right\}_{0}^{\infty}$ is the sequence of Fourier cosine coefficients of a function $F$ also in $L^{p}(\omega)$; moreover, there is a constant $c$ independent of $f$ such that $\|F\|_{p, \omega} \leq c\|f\|_{p, \omega}$.

If $\omega$ satisfies the $A_{p}$ condition, then as we shall show, (1.3) is satisfied so that we have the following corollary.

Corollary 2. If $1<p<\infty$ and $\omega$ satisfies the $A_{p}$ condition then the conclusion of Theorem 2 holds.

Concerning the sequences $\left\{A_{\nu}^{\prime}\right\}$ we have the following results.

TheOREM 3. Let $p=1$. Suppose $\omega$ satisfies (1.1) and there is a constant $C$ such that for almost all $t, 0<t<\pi$,

$$
\int_{t}^{\pi} \omega(x) \frac{d x}{x} \leq C \omega(t) .
$$

If $\left\{a_{\nu}\right\}_{0}^{\infty}$ is the sequence of Fourier cosine coefficients of an $f \in L^{1}(\omega)$ then $\left\{A_{\nu}^{\prime}\right\}_{0}^{\infty}$ is the sequence of Fourier cosine coefficients of a function $F$ also in $L^{1}(\omega)$; moreover, there is a constant $c$ independent of $f$ such that $\|F\|_{1, \omega} \leq$ $c\|f\|_{1, \omega}$. 
THEOREM 4. Let $1<p<\infty$. Suppose $\omega$ satisfies (1.1) and that there is a constant $C$ such that for some $\varepsilon>0$

$$
\left(\int_{r}^{\pi}\left(\frac{r}{x}\right)^{\varepsilon} x^{-p} \omega(x) d x\right)^{1 / p}\left(\int_{0}^{r} \omega(x)^{-1 /(p-1)} d x\right)^{1 / p^{\prime}} \leq C
$$

holds for all $0<r<\pi$. If $\left\{a_{\nu}\right\}_{0}^{\infty}$ is the sequence of Fourier cosine coefficients of an $f \in L^{p}(\omega)$ then $\left\{A_{\nu}^{\prime}\right\}_{0}^{\infty}$ is the sequence of Fourier cosine coefficients of a function $F$ also in $L^{p}(\omega)$; moreover, there is a constant $c$ independent of $f$ such that $\|F\|_{p, \omega} \leq c\|f\|_{p, \omega}$.

As Bellman [3] pointed out, there is a certain 'duality' between the Theorems for $\left\{A_{\nu}\right\}$ and $\left\{A_{\nu}^{\prime}\right\}$. His duality Theorem may be generalized as follows. For a sequence $\left\{b_{\nu}\right\}_{0}^{\infty}$ let $B_{0}^{\prime}=b_{0}$ and $B_{\nu}^{\prime}=\sum_{j=\nu}^{\infty} b_{j} / j$ for $\nu=$ $1,2, \ldots$

TheOREM 5. Let $1<p<\infty$. Suppose $\omega$ satisfies (1.1) and that there is a constant $C$ such that for some $\varepsilon>0$

$$
\left(\int_{0}^{r} \omega(x) d x\right)^{1 / p}\left(\int_{r}^{\pi}\left(\frac{r}{x}\right)^{\varepsilon} x^{-p^{\prime}} \omega(x)^{-1 /(p-1)} d x\right)^{1 / p^{\prime}} \leq C
$$

holds for $0<r<\pi$. If $\left\{a_{\nu}\right\}_{0}^{\infty}$ and $\left\{b_{\nu}\right\}_{0}^{\infty}$ are the sequences of Fourier cosine coefficients of functions $f \in L^{p}(\omega)$ and $g \in L^{p^{\prime}}\left(\omega^{-1 /(p-1)}\right)$ respectively, then $\left\{A_{\nu}\right\}_{0}^{\infty}$ and $\left\{B_{\nu}^{\prime}\right\}_{0}^{\infty}$ are the sequences of Fourier cosine coefficients of functions $F \in L^{p}(\omega)$ and $G \in L^{p^{\prime}}\left(\omega^{-1 /(p-1)}\right)$ respectively which satisfy the identity

$$
\int_{0}^{\pi}\{f(x) G(x)-F(x) g(x)\} d x=0 .
$$

The proofs will depend on the following Lemma which is of interest in its own right.

Lemma. Let $1 \leq p<\infty$ and suppose $\omega$ satisfies (1.1). If $\left\{a_{\nu}\right\}_{0}^{\infty}$ is the sequence of Fourier cosine coefficients of a function $f \in L^{p}(\omega)$, then $\left\{c_{\nu}\right\}_{0}^{\infty}$ given by $c_{0}=0, c_{\nu}=a_{\nu} / \nu, \nu=1,2, \ldots$ is the sequence of Fourier cosine coefficients of a function $H$ also in $L^{p}(\omega)$; moreover, there is a constant $c$ independent of $f$ such that $\|H\|_{p, \omega} \leq c\|f\|_{p, \omega}$.

2. Proof of the lemma. According to $[8$, p. 180] the function $H$ is given by

$$
H(x)=\frac{1}{\pi} \int_{-\pi}^{\pi} f(x+t) \log \left(\frac{1}{2|\sin (t / 2)|}\right) d t, \quad 0<x<\pi .
$$


We shall carry out the proof assuming that $1<p<\infty$; the required modifications for the case $p=1$ will be self-evident.

Consider first $H_{1}(x)$ where $H(x)=H_{1}(x)+H_{2}(x)$ with

$$
H_{1}(x)=\frac{1}{\pi} \int_{\pi \geq|t| \geq \pi / 3} f(x+t) \log \left(\frac{1}{2|\sin (t / 2)|}\right) d t .
$$

From Hölder's inequality and the periodic property of $f$ and $\omega$ it follows that

$$
\begin{aligned}
\left|H_{1}(x)\right| \leq & \left(\frac{\log 2}{\pi}\right) \int_{\pi \geq|t| \geq \pi / 3}|f(x+t)| d t \\
\leq & \left(\frac{\log 2}{\pi}\right)\left(\int_{\pi \geq|t| \geq \pi / 3}|f(x+t)|^{p} \omega(x+t) d t\right)^{1 / p} \\
& \times\left(\int_{\pi \geq|t| \geq \pi / 3} \omega(x+t)^{-1 /(p-1)} d t\right)^{1 / p^{\prime}} \\
\leq & \left(\frac{2 \log 2}{\pi}\right)\left(\int_{0}^{\pi}|f(t)|^{p} \omega(t) d t\right)^{1 / p}\left(\int_{0}^{\pi} \omega(t)^{-1 /(p-1)} d t\right)^{1 / p^{\prime}}
\end{aligned}
$$

and hence

$$
\left(\int_{0}^{\pi}\left|H_{1}(x)\right|^{p} \omega(x) d x\right)^{1 / p} \leq \frac{2 \log 2}{\pi} \theta(\pi)\left(\int_{0}^{\pi}|f(t)|^{p} \omega(t) d t\right)^{1 / p}
$$

Now to treat $H_{2}(x)$, observe first that for fixed $u, 0<u<1$, we have

$$
\begin{aligned}
\int_{0}^{\pi} \omega(x)\left[\int_{|t| \leq \pi u / 2}|f(x+t)| d t\right]^{p} d x \\
\quad \leq 4\left[\theta\left(\frac{3 \pi u}{2}\right)\right]^{p} \int_{0}^{\pi}|f(t)|^{p} \omega(t) d t .
\end{aligned}
$$

To see this, choose the integer $N$ so that $N u \geq 2$, and let, for convenience, $a=\pi u / 2$. Then the left side of (2.2) is bounded above by

$$
\sum_{k=1}^{N} \int_{(k-1) a}^{k a} \omega(x)\left[\int_{|t| \leq a}|f(x+t)| d t\right]^{p} d x
$$


Enlarging the inner integral and applying Hölder's inequality shows this is further bounded by

$$
\begin{aligned}
\sum_{k=1}^{N} \int_{(k-1) a}^{k a} \omega(x)\left[\int_{(k-2) a}^{(k+1) a}|f(t)| d t\right]^{p} d x & \\
\leq & \sum_{k=1}^{N} \int_{(k-1) a}^{k a} \omega(x) d x\left[\int_{(k-2) a}^{(k+1) a} \omega(t)^{-1 /(p-1)} d t\right]^{p-1} \\
& \times\left[\int_{(k-2) a}^{(k+1) a}|f(t)|^{p} \omega(t) d t\right] \\
\leq & {[\theta(3 a)]^{p} \sum_{k=1}^{N} \int_{(k-2) a}^{(k+1) a}|f(t)|^{p} \omega(t) d t }
\end{aligned}
$$

where to obtain the last inequality we have used the definition of $\theta$. Finally, since the intervals $((k-2) a,(k+1) a)$ have limited overlap, we obtain (2.2).

Returning to $H_{2}(x)$ we have

$$
\begin{aligned}
\left|H_{2}(x)\right| & \leq \frac{1}{\pi} \int_{-\pi / 3}^{\pi / 3}|f(x+t)|\left(\int_{2|\sin (t / 2)|}^{1} \frac{d u}{u}\right) d t \\
& \leq \frac{1}{\pi} \int_{-\pi / 3}^{\pi / 3}|f(x+t)|\left(\int_{2|t| / \pi}^{1} \frac{d u}{u}\right) d t \\
& \leq \frac{1}{\pi} \int_{0}^{1} \frac{d u}{u} \int_{|t| \leq \pi u / 2}|f(x+t)| d t
\end{aligned}
$$

by an appeal to Fubini's Theorem. Minkowski's inequality for integrals followed by (2.2) then yields

$$
\begin{aligned}
\left(\int_{0}^{\pi} \mid H_{2}(x)\right. & \mid p \omega(x) d x)^{1 / p} \\
\leq & \frac{1}{\pi} \int_{0}^{1} \frac{d u}{u}\left\{\int_{0}^{\pi} \omega(x)\left[\int_{|t| \leq \pi u / 2}|f(x+t)| d t\right]^{p} d x\right\}^{1 / p} \\
\leq & \frac{4^{1 / p}}{\pi}\left(\int_{0}^{1} \theta\left(\frac{3 \pi u}{2}\right) \frac{d u}{u}\right)\left(\int_{0}^{\pi}|f(t)|^{p} \omega(t) d t\right)^{1 / p} .
\end{aligned}
$$

A change of variable in the first integral on the right shows, in view of (1.1), that

$$
\left(\int_{0}^{\pi}\left|H_{2}(x)\right|^{p} \omega(x) d x\right)^{1 / p} \leq c\left(\int_{0}^{\pi}|f(t)|^{p} \omega(t) d t\right)^{1 / p} .
$$

This, together with (2.1), completes the proof of the lemma. 
3. Proof of Theorem 1. Assume first that $a_{0}=0$. Then as Hardy [4] has shown, $F$ is given by $F(x)=\left[F_{1}(x)+H(x)\right] / 2$ where $H$ is the function of the Lemma and $F_{1}(x)=\int_{x}^{\pi} f(t) \cot (t / 2) d t$. Thus it suffices to prove that $\left\|F_{1}\right\|_{1, \omega} \leq c\|f\|_{1, \omega}$. To see this, observe that $\cot (t / 2) \leq 2 / t$ so that

$$
\begin{aligned}
\int_{0}^{\pi}\left|F_{1}(x)\right| \omega(x) d x & \leq 2 \int_{0}^{\pi} \omega(x)\left(\int_{x}^{\pi}|f(t)| \frac{d t}{t}\right) d x \\
& =2 \int_{0}^{\pi}|f(t)|\left(\frac{1}{t} \int_{0}^{t} \omega(x) d x\right) d t \\
& \leq 2 C \int_{0}^{\pi}|f(t)| \omega(t) d t
\end{aligned}
$$

by Fubini's Theorem and the hypothesis (1.2).

If now $a_{0} \neq 0$, the above argument shows that there is a function $F(x)$ with Fourier cosine coefficients $\left\{A_{\nu}\right\}_{0}^{\infty}$ and which satisfies $\left\|F-a_{0} / 2\right\|_{1, \omega} \leq c\left\|f-a_{0} / 2\right\|_{1, \omega}$. Now the triangle inequality and the observation

$$
\begin{aligned}
\left\|a_{0} / 2\right\|_{1, \omega} & =\left(\int_{0}^{\pi} \omega(x) d x\right)\left|\frac{1}{\pi} \int_{0}^{\pi} f(t) d t\right| \\
& \leq \frac{1}{\pi}\left(\int_{0}^{\pi} \omega(x) d x\right)\left(\int_{0}^{\pi}|f(t)| \omega(t) d t\right) \underset{(0, \pi)}{\left.\operatorname{ess} \cdot \sup _{(1, \omega} 1 / \omega(t)\right)} \\
& \leq \frac{1}{\pi} \theta(\pi)\|f\|_{1, \omega}
\end{aligned}
$$

shows that $\|F\|_{1, \omega} \leq c\|f\|_{1, \omega}$ for some constant $c$. This completes the proof of Theorem 1 .

4. Proof of Theorem 2 and Corollary 2. We prove the Theorem first. Just as in the proof of Theorem 1 , we may assume that $a_{0}=0$ for the general case follows easily from this, and it therefore suffices to prove that

$$
\int_{0}^{\pi} \omega(x)\left|\int_{x}^{\pi} f(t) \frac{d t}{t}\right|^{p} d x \leq c \int_{0}^{\pi}|f(t)|^{p} \omega(t) d t
$$

for some constant $c$. According to [6] (or [2]) a sufficient (and necessary) condition for (4.1) to hold is that

$$
\left(\int_{0}^{r} \omega(x) d x\right)^{1 / p}\left(\int_{r}^{\pi} x^{-p^{\prime}} \omega(x)^{-1 /(p-1)} d x\right)^{1 / p^{\prime}} \leq C
$$

for all $0<r<\pi$. Since Lemma 2 of [2] shows that (4.2) and (1.3) are equivalent, the proof is complete. 
To prove the Corollary, we shall show that (1.3) holds if $\theta(u) \leq C u$. To see this, note that the definition of $\theta(t)$ yields

$$
\left(\int_{0}^{r} \omega(x) d x\right)^{p^{\prime} / p}\left(\int_{r}^{t} \omega(x)^{-1 /(p-1)} d x\right) \leq C^{p^{\prime}} t^{p^{\prime}}
$$

for $0<r<t<\pi$. Multiplying this by $(r / t)^{\varepsilon} t^{-p^{\prime}-1}$ and integrating the result over $r<t<\pi$ leads by Fubini's Theorem to

$$
\begin{aligned}
\left(\int_{0}^{r} \omega(x) d x\right)^{p^{\prime} / p} \int_{r}^{\pi} \omega(x)^{-1 /(p-1)}\left\{\left(\frac{r}{x}\right)^{\varepsilon} x^{-p^{\prime}}-\left(\frac{r}{\pi}\right)^{\varepsilon} \pi^{-p^{\prime}}\right\} d x \\
\leq\left(p^{\prime}+\varepsilon\right) C^{p^{\prime}} \int_{r}^{\pi}\left(\frac{r}{t}\right)^{\varepsilon} \frac{d t}{t} \leq\left(p^{\prime}+\varepsilon\right) \varepsilon^{-1} C^{p^{\prime}} .
\end{aligned}
$$

Transposing the negative term on the left side and dominating it in terms of $\theta(\pi)$ shows that (1.3) holds. This proves the Corollary.

5. Proof of Theorem 3. Observe first that (1.4) and Fubini's Theorem shows that

$$
\begin{aligned}
\int_{0}^{\pi} \omega(x)\left|\frac{1}{x} \int_{0}^{x} f(t) d t\right| d x & \leq \int_{0}^{\pi}|f(t)| d t \int_{t}^{\pi} \frac{\omega(x)}{x} d x \\
& \leq C \int_{0}^{\pi}|f(t)| \omega(t) d t .
\end{aligned}
$$

Hence, if $f \in L^{1}(\omega)$,

$$
\begin{aligned}
\int_{0}^{\pi} & f(t)\left|\log (\pi / t) d t=\int_{0}^{\pi}\right| f(t) \mid\left(\int_{t}^{\pi} \frac{d x}{x}\right) d t \\
& =\int_{0}^{\pi}\left(\frac{1}{x} \int_{0}^{x}|f(t)| d t\right) d x \\
& \leq\left(\int_{0}^{\pi} \omega(x)\left(\frac{1}{x} \int_{0}^{x}|f(t)| d t\right) d x\right)(\underset{(0, \pi)}{\operatorname{ess} \cdot \sup 1 / \omega(x))} \\
& \leq C\left(\int_{0}^{\pi}|f(t)| \omega(t) d t\right)(\underset{(0, \pi)}{\operatorname{ess} \cdot \sup 1 / \omega(x))} \\
& <\infty .
\end{aligned}
$$

Now, if $a_{0}=0$, Loo [5, pp. 272-274] has shown that (5.2) ensures that $F(x)$ is given by

$$
F(x)=\left(\cot (x / 2) \int_{0}^{x} f(t) d t+H(x)\right) / 2
$$

where $H(x)$ is given by the Lemma. Hence (5.1) and the inequality $\cot (x / 2) \leq \pi / x$ show that $F \in L^{1}(\omega)$. This proves the Theorem for the case $a_{0}=0$, and as before, the general case follows easily from this. 
6. Proof of Theorem 4. According to [6] and Lemma 2 of [2], the hypothesis (1.5) ensures that

$$
\int_{0}^{\pi} \omega(x)\left[\frac{1}{x} \int_{0}^{x}|f(t)| d t\right]^{p} d x \leq c \int_{0}^{\pi}|f(t)|^{p} \omega(t) d t .
$$

Hence, Fubini's Theorem and Hölder's inequality shows that

$$
\begin{aligned}
& \int_{0}^{\pi}|f(t)| \log (\pi / t) d t=\int_{0}^{\pi}\left(\frac{1}{x} \int_{0}^{x}|f(t)| d t\right) d x \\
& \quad \leq\left(\int_{0}^{\pi} \omega(x)\left[\frac{1}{x} \int_{0}^{x}|f(t)| d t\right]^{p} d x\right)^{1 / p}\left(\int_{0}^{\pi} \omega(x)^{-1 /(p-1)} d x\right)^{1 / p^{\prime}} \\
& \quad \leq c\left(\int_{0}^{\pi}|f(t)|^{p} \omega(t) d t\right)^{1 / p}\left(\int_{0}^{\pi} \omega(x)^{-1 /(p-1)} d x\right)^{1 / p^{\prime}}<\infty
\end{aligned}
$$

whenever $f \in L^{p}(\omega)$. Hence, if $a_{0}=0, F(x)$ is again given by (5.3) and (6.1) shows that $F \in L^{p}(\omega)$. The general case follows easily from this.

7. Proof of Theorem 5. The hypothesis and Theorem 2 show that there is $F \in L^{p}(\omega)$ with Fourier cosine coefficients $\left\{A_{\nu}\right\}_{0}^{\infty}$ satisfying

$$
\int_{0}^{\pi}|F(x)|^{p} \omega(x) d x \leq c \int_{0}^{\pi}|f(x)|^{p} \omega(x) d x .
$$

Further, since $\theta\left(\omega^{-1 /(p-1)}, p^{\prime} ; u\right)=\theta(\omega, p ; u)$, the hypothesis and Theorem 4 yields a $G \in L^{p^{\prime}}\left(\omega^{-1 /(p-1)}\right)$ with Fourier cosine coefficients $\left\{B_{\nu}^{\prime}\right\}_{0}^{\infty}$ satisfying

$$
\int_{0}^{\pi}|G(x)|^{p^{\prime}} \omega(x)^{-1 /(p-1)} d x \leq c \int_{0}^{\pi}|g(x)|^{p^{\prime}} \omega(x)^{-1 /(p-1)} d x .
$$

If the left side of (1.7) is denoted by $L(f, g)$, Hölder's inequality followed by (7.1) and (7.2) then shows that $L$ is a bilinear functional on $L^{p}(\omega) \times$ $L^{p^{\prime}}\left(\omega^{-1 /(p-1)}\right)$ satisfying

$$
|L(f, g)| \leq c\|f\|_{p, \omega}\|g\|_{p^{\prime}, \omega^{-1 /(p-1)}} .
$$

A direct computation (or an appeal to Bellman's Theorem [3]) shows that $L(f, g)=0$ whenever $f$ and $g$ belong to the class $\mathscr{P}$ of finite linear combinations of $\{\cos \nu x\}_{0}^{\infty}$. Choose $f_{n}, g_{n} \in \mathcal{P}$ with $\left\|f_{n}-f\right\|_{p, \omega} \rightarrow 0$ and $\left\|g_{n}-g\right\|_{p^{\prime}, \omega^{-1 /(p-1)}} \rightarrow 0$ as $n \rightarrow \infty$ (see [7, p. 89]). Then

$$
\begin{aligned}
L(f, g) & =\left[L(f, g)-L\left(f, g_{n}\right)\right]+\left[L\left(f, g_{n}\right)-L\left(f_{n}, g_{n}\right)\right] \\
& =L\left(f, g-g_{n}\right)+L\left(f-f_{n}, g_{n}\right)
\end{aligned}
$$


so that (7.3) yields

$$
|L(f, g)| \leq c\left\{\|f\|_{p, \omega}\left\|g-g_{n}\right\|_{p^{\prime}, \omega^{-1 /(p-1)}}+\left\|f-f_{n}\right\|_{p, \omega}\left\|g_{n}\right\|_{p^{\prime}, \omega^{-1 /(p-1)}}\right\}
$$

and since the right side tends to zero as $n \rightarrow \infty$, it follows that $L(f, g)=0$ and the Theorem is proved.

\section{REFERENCES}

1. K. F. Andersen, On the transformation of Fourier coefficients of certain classes of functions, Pacific J. Math., (to appear).

2. K. F. Andersen and B. Muckenhoupt, Weighted weak type Hardy inequalities with applications to maximal functions and Hilbert transforms, Studia Math., 72 (1981), 9-26.

3. R. Bellman, A note on a theorem of Hardy on Fourier coefficients, Bull. Amer. Math. Soc., 50 (1944), 741-744.

4. G. H. Hardy, Notes on some points in the integral calculus LXVI, Messenger Math., 58 (1928), 50-52.

5. C.-T. Loo, Notes on the properties of Fourier coefficients, Amer. J. Math., 71 (1949), 269-282.

6. B. Muckenhoupt, Hardy's inequality with weights, Studia Math., 44 (1972), 31-38.

7. W. Rudin, Real and Complex Analysis, McGraw Hill, 1966.

8. A. Zygmund, Trigonometric Series, Vol. I and II combined, Cambridge Univ. Press, 1968.

Received August 19, 1981. Research supported in part by NSERC Grant \#A-8185.

UNIVERSITY OF ALBERTA

Edmonton, Alberta, CANAda 


\section{PACIFIC JOURNAL OF MATHEMATICS}

EDITORS

Donald BABBITT (Managing Editor)

University of California

Los Angeles, CA 90024

Hugo Rossi

University of Utah

Salt Lake City, UT 84112

C. C. Moore and Arthur Ogus

University of California

Berkeley, CA 94720
J. DugunduI

Department of Mathematics

University of Southern California

Los Angeles, CA 90089-1113

R. FinN and H. SAmelson

Stanford University

Stanford, CA 94305

\section{ASSOCIATE EDITORS}
R. ARENS
E. F. BECKENBACH
B. H. NEUMANN
F. WolF
K. YoshidA (1906-1982)

\section{SUPPORTING INSTITUTIONS}

UNIVERSITY OF ARIZONA

UNIVERSITY OF BRITISH COLUMBIA

CALIFORNIA INSTITUTE OF TECHNOLOGY

UNIVERSITY OF CALIFORNIA

MONTANA STATE UNIVERSITY

UNIVERSITY OF NEVADA, RENO

NEW MEXICO STATE UNIVERSITY

OREGON STATE UNIVERSITY
UNIVERSITY OF OREGON

UNIVERSITY OF SOUTHERN CALIFORNIA

STANFORD UNIVERSITY

UNIVERSITY OF HAWAII

UNIVERSITY OF TOKYO

UNIVERSITY OF UTAH

WASHINGTON STATE UNIVERSITY

UNIVERSITY OF WASHINGTON 


\section{Pacific Journal of Mathematics}

Vol. 105, No. 1 September, 1983

Kenneth F. Andersen, On the transformation of Fourier coefficients of

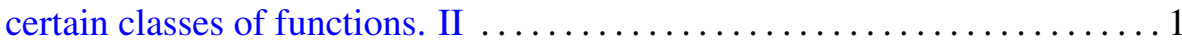

Gavin Brown, Irving Leonard Glicksberg and Edwin Hewitt, Indicator functions with large Fourier transforms $\ldots \ldots \ldots \ldots \ldots \ldots \ldots \ldots \ldots \ldots$

Shih-Sen Chang, Some random fixed point theorems for continuous random

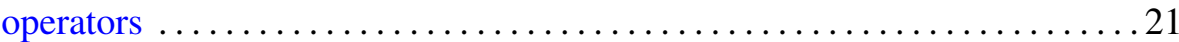

K. C. Chattopadhyay and Olav Njstad, Quasiregular nearness spaces and

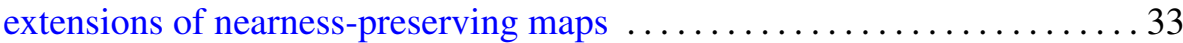

Thomas W. Cusick, The two-dimensional Diophantine approximation

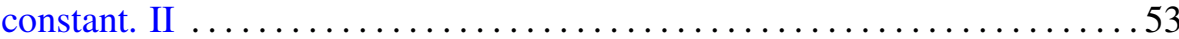

Eric Karel van Douwen and Jan van Mill, Spaces without remote points . . .669 Hector O. Fattorini, Convergence and approximation theorems for

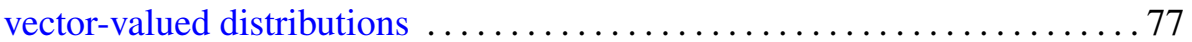

John J. F. Fournier and Louis Pigno, Analytic and arithmetic properties of

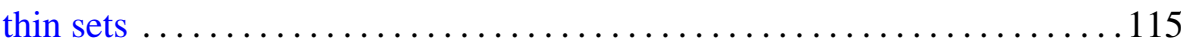

David Goss, On a new type of $L$-function for algebraic curves over finite

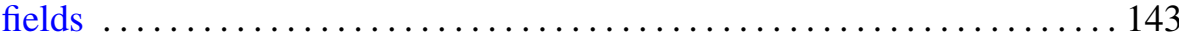

Douglas Austin Hensley, Lattice vertex polytopes with interior lattice

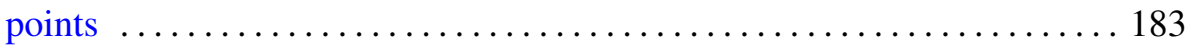

J. K. Kohli, Monotone extensions of mappings and their applications ...... 193

John C. Morgan, II, On equivalent category bases . . . . . . . . . . . . 207

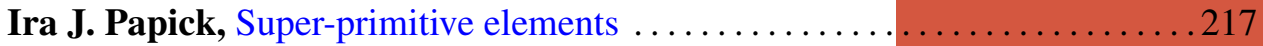

José Luis Rubio de Francia and José Luis Torrea, Vector extensions of

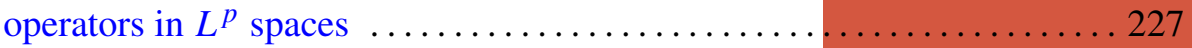

Mark Phillip Thomas, Closed ideals of $l^{1}\left(\omega_{n}\right)$ when $\left\{\omega_{n}\right\}$ is star-shaped $\ldots 237$ 